\title{
OSCILLATION AND NONOSCILLATION OF NONLINEAR NEUTRAL DELAY DIFFERENCE EQUATIONS
}

\section{IIIIII}

\author{
E. THANDAPANI AND P. MOHAN KUMAR
}

\begin{abstract}
In this paper, the authors establish some sufficient conditions for oscillation and nonoscillation of the second order nonlinear neutral delay difference equation

$$
\Delta^{2}\left(x_{n}-p_{n} x_{n-k}\right)+q_{n} f\left(x_{n-\ell}\right)=0, \quad n \geq n_{0}
$$

where $\left\{p_{n}\right\}$ and $\left\{q_{n}\right\}$ are non-negative sequences with $0<p_{n} \leq 1$, and $k$ and $\ell$ are positive integers.
\end{abstract}

\section{Introduction}

Consider the second order nonlinear neutral delay difference equation

$$
\Delta^{2}\left(x_{n}-p_{n} x_{n-k}\right)+q_{n} f\left(x_{n-\ell}\right)=0, \quad n \geq n_{0} \in \mathbb{N}
$$

where $\mathbb{N}=\{0,1,2, \ldots\}$ and $\Delta$ is the forward difference operator defined by $\Delta x_{n}=$ $x_{n+1}-x_{n}$, subject to the following conditions:

$\left(c_{1}\right)\left\{p_{n}\right\}$ and $\left\{q_{n}\right\}$ are non-negative real sequences with $\left\{q_{n}\right\}$ not identically zero for infinitely many values of $n$;

$\left(c_{2}\right) f: \mathbb{R} \rightarrow \mathbb{R}$ is continuous and nondecreasing such that $u f(u)>0$ for $u \neq 0$;

$\left(c_{3}\right)$ there is a positive constant $p$ such that $0<p_{n} \leq p<1$, and $k$ and $\ell$ are positive integers.

For any real sequence $\left\{\phi_{n}\right\}$ defined in $n_{0}-\theta \leq n \leq n_{0}$ where $\theta=\max \{k, \ell\}$, equation (11) has a solution $\left\{x_{n}\right\}$ defined for $n \geq n_{0}$ and satisfying the initial condition $x_{n}=\phi_{n}$ for $n_{0}-\theta \leq n \leq n_{0}$. A solution $\left\{x_{n}\right\}$ of equation (11) is oscillatory if it is neither eventually positive nor eventually negative, and nonoscillatory otherwise.

In several recent papers $[3,4,7-20]$, the oscillatory and nonoscillatory behavior of solutions of equation (11) has been studied when $\left\{p_{n}\right\}$ is a non-positive real sequence. However in [14], the authors consider the case $\left\{p_{n}\right\}$ is non-negative and attempted to extend the known results in [1] on delay difference equation to neutral difference equation with $p_{n} \equiv p \in(0,1)$. In fact the authors [14] proved the following two theorems:

Received September 6, 2006; revised March 1, 2007.

2000 Mathematics Subject Classification. 39A10.

Key words and phrases. Neutral difference equation, second order, superlinear, sublinear, oscillation, nonoscillation. 
Theorem A. Assume that $0<p<1,\left\{q_{n}\right\}$ is a nonnegative real sequence and $f: \mathbb{R} \rightarrow \mathbb{R}$ is continuous and nondecreasing with $u f(u)>0$ for $u \neq 0$. If

$$
0<\int_{\varepsilon}^{\infty} \frac{d x}{f(x)}, \int_{-\infty}^{-\varepsilon} \frac{d x}{f(x)}<\infty \text { for all } \varepsilon>0
$$

then every solution of the equation

$$
\Delta^{2}\left(x_{n}-p x_{n-k}\right)+q_{n} f\left(x_{n-\ell}\right)=0, \quad n \geq n_{0}
$$

is oscillatory if and only if

$$
\sum_{n=n_{0}}^{\infty} n q_{n}=\infty
$$

Theorem B. Assume that $0<p<1,\left\{q_{n}\right\}$ is a nonegative real sequence and $f$ : $\mathbb{R} \rightarrow \mathbb{R}$ is continuous and nondecreasing with $u f(u)>0$ for $u \neq 0$. If

$$
0<\int_{0}^{\varepsilon} \frac{d x}{f(x)}, \int_{-\varepsilon}^{0} \frac{d x}{f(x)}<\infty \text { for all } \varepsilon>0
$$

and

$$
f(u v) \geq f(u) f(v) \text { if } u v>0 \text { and }|v| \geq M
$$

for some constant $M>0$, then every solution of equation (3) is oscillatory if and only if

$$
\sum_{n=n_{0}}^{\infty} f(n) q_{n}=\infty
$$

In the following we give an example which illustrates the sufficient part of Theorem A is false.

Let $k, \ell \geq 1,0<p<1, \alpha>1$. Choose $\lambda>-\frac{1}{k} \log p$ and set $q_{n}=\frac{\left(p e^{\lambda k}-1\right)\left(e^{-\lambda}-1\right)^{2} e^{(\alpha-1) \lambda n}}{e^{\lambda \ell k}}$. It is easy to see that $\left\{x_{n}\right\}=\left\{e^{-\lambda n}\right\}$ is a positive solution of the equation

$$
\Delta^{2}\left(x_{n}-p x_{n-k}\right)+q_{n}\left|x_{n-\ell}\right|^{\alpha-1} x_{n-\ell}=0, \quad n \geq n_{0}
$$

even if (4) is satisfied. The error occurred in the proof is due to their false assertion that if $\left\{x_{n}\right\}$ is eventually positive solution of equation (3) then $z_{n}=x_{n}-p x_{n-k}$ is also eventually positive. The same false assertion was also used in the proof of Theorem B and therefore the sufficient part of Theorem B may not be true. Therefore, so far there are hardly any results on the oscillatory behavior of solutions of equation (1) with $\left\{p_{n}\right\}$ is nonnegative.

In this paper, we study the oscillatory and nonoscillatory behavior of equation (1) with $0 \leq p_{n}<1$ and the nonlinear function $f$ is either supelinear or sublinear. In Section 2 , we present a new sufficient condition for the oscillation of all solutions of equation (1) 
when $f$ is superlinear and extend the necessary part of Theorem A to equation (11). Section 3 contains similar results for equation (11) when $f$ is sublinear. For basic results on the oscillation theory of difference equations one can refer the recent monographs [1] and [2].

\section{Oscillation results for superlinear case}

In this section we shall investigate the oscillatory behavior of solutions of equation (11) when $f$ is superlinear. The function $f$ is said to be superlinear if there exists a constant $\alpha>0$ such that

$$
\lim _{x \rightarrow 0} \inf \left(\frac{|f(x)|}{|x|^{\alpha}}\right)>0 .
$$

We need the following lemma given in [12] to prove our main result of this section.

Lemma 1. Let $\left\{Q_{n}\right\}$ be a nonnegative real sequence, $f: \mathbb{R} \rightarrow \mathbb{R}$ be continuous with $u f(u)>0$ for $u \neq 0$, and $\delta$ be a positive integer. Assume that there exist $\beta>0$ and $\lambda>\frac{1}{\delta} \log \beta$ such that $\lim _{x \rightarrow 0}\left(\frac{|f(x)|}{|x|^{\beta}}\right)>0$ and $\lim _{n \rightarrow \infty} \inf \left[Q_{n} \exp \left(-e^{\lambda n}\right)\right]>0$ then the following inequality

$$
\Delta x_{n}+Q_{n} f\left(x_{n-\delta}\right) \leq 0, \quad n \geq n_{0},
$$

has no eventually positive solutions.

Theorem 2. With respect to the difference equation (11) assume that $\ell>k$, and condition (9) hold. If there exist a $\lambda>\frac{\log \alpha}{\ell-k}$ such that

$$
\lim _{n \rightarrow \infty} \inf q_{n} \exp \left(-e^{\lambda n}\right)>0
$$

then every solution of equation (11) is oscillatory.

Proof. Let $\left\{x_{n}\right\}$ be a nonoscillatory solution of equation (11). We may assume without loss of generality that $x_{n}>0$ and $0<p_{n}<p$ for all $n \geq n_{0}$ for some integer $n_{0} \in N$. Set

$$
y_{n}=x_{n}-p_{n} x_{n-k} .
$$

Then it follows from equation (11) that $\Delta^{2} y_{n} \leq 0$ for all $n \geq n_{0}+\theta$. This implies that $\left\{\Delta y_{n}\right\}$ is nonincreasing for all $n \geq n_{0}+\theta$. Hence, there are two possible cases that $\Delta y_{n}>0$ for all $n \geq n_{0}+\theta$ or $\Delta y_{n}<0$ for all $n \geq n_{1}$ for some integer $n_{1} \geq n_{0}$. If the later case holds, then there exits a constant $c>0$ and an integer $n_{2} \geq n_{1}$ such that

$$
x_{n}-p_{n} x_{n-k} \leq-c, \quad n \geq n_{2},
$$

which implies that

$$
x_{n} \leq-c+p x_{n-k}, \quad n \geq n_{2} .
$$


From (12), we have

$$
\begin{aligned}
x_{n_{2}+k} & \leq-c+p x_{n_{2}} \\
x_{n_{2}+2 k} & \leq-c+p\left(x_{n_{2}+k}\right) \leq-c-p c+p^{2} x_{n_{2}} \\
x_{n_{2}+3 k} & \leq-c+p\left(x_{n_{2}+2 k}\right) \leq-c-p c-p^{2} c+p^{3} x_{n_{2}}
\end{aligned}
$$

and hence it follows that

$$
x_{n_{2}+j k} \leq-\sum_{i=0}^{j-1} c p^{i}+p^{j} x_{n_{2}},
$$

and so $x_{n_{2}+j k}<0$ for large $j$, which contradicts the fact that $x_{n}>0$ for all $n \geq n_{0}$. Hence

$$
\Delta y_{n}>0 \text { for all } n \geq n_{0}+\theta \text {. }
$$

From (13), it follows that $\left\{y_{n}\right\}$ is increasing for all $n \geq n_{0}+\theta$ and so there are two possible cases:

(i) $y_{n}<0$ for $n \geq n_{0}+\theta$ or

(ii) $y_{n}>0$ for $n \geq n_{3}$ for integer $n_{3} \geq n_{2}$.

If case (i) holds, that is, $y_{n}<0$ for all $n \geq n_{0}+\theta$ then

$$
x_{n-\ell}>-\frac{1}{p} y_{n+k-\ell}, \quad n \geq n_{0}+2 \theta,
$$

and

$$
\Delta^{2} y_{n}+q_{n} f\left(-\frac{1}{p} y_{n+k-\ell}\right) \leq 0, \quad n \geq n_{0}+2 \theta
$$

Summing the inequality (15) from $n \geq n_{0}+2 \theta$ to $\infty$, we find

$$
-\Delta y_{n}+\sum_{s=n}^{\infty} q_{s} f\left(-\frac{1}{p} y_{s+k-\ell}\right) \leq 0, \quad n \geq n_{0}+2 \theta .
$$

From the assumption $\lambda>\frac{\log \alpha}{\ell-k}$, we can choose an integer $m$ such that $1 \leq m \leq \ell-k$ and

$$
\alpha e^{-\lambda(\ell-k-m)}<1
$$

Note that $-\Delta y_{n}$ is decreasing for all $n \geq n_{0}+\theta$, it follows from (16) that

$$
-\Delta y_{n}+\left(\sum_{s=n}^{n+m} q_{s}\right) f\left(-\frac{1}{p} y_{n+k-\ell+m}\right) \leq 0, \quad n \geq n_{0}+2 \theta .
$$

Set

$$
z_{n}=-\frac{1}{p} \Delta y_{n}, \quad \delta=\ell-k-m, \quad Q_{n}=\frac{1}{p} \sum_{s=n}^{n+m} q_{s} .
$$

Then (18) can be written as

$$
\Delta z_{n}+Q_{n} f\left(z_{n-\delta}\right) \leq 0, \quad n \geq n_{0}+2 \theta .
$$


This shows that (19) has an eventually positive solution $\left\{z_{n}\right\}$. On the other hand, by (10),

$$
\lim _{n \rightarrow \infty} \inf \left[Q_{n} \exp \left(-e^{\lambda n}\right)\right] \geq \frac{(m+1)}{p} \lim _{n \rightarrow \infty} \inf \left[\left(\min _{n \leq s \leq n+m} q_{s}\right) \exp \left(-e^{\lambda n}\right)\right]>0 .
$$

In view of (17) and (20), Lemma 1 implies that the inequality (19) has no eventually positive solutions. This contradiction shows that case (i) is impossible.

If case (ii) holds, that is , $y_{n}>0$ for all $n \geq n_{3}$, then it follows from equation (11) that

$$
\Delta^{2} y_{n}+q_{n} f\left(y_{n-\ell}\right) \leq 0, \quad n \geq n_{3}+\theta .
$$

Summing (21) from $n_{4}=n_{3}+\theta$ to $n$ and then taking $n \rightarrow \infty$, we find

$$
\sum_{n=n_{4}}^{\infty} q_{n} f\left(y_{n-\ell}\right) \leq \Delta y_{n_{4}} .
$$

Since $f\left(y_{n}\right)$ is nondecreasing for all $n \geq n_{4}$, it follows from (22) that

$$
f\left(y_{n_{3}}\right) \sum_{s=n}^{\infty} q_{s} \leq \Delta y_{n_{4}}<\infty
$$

which contradicts (10) and so case (ii) is also impossible. This completes the proof of the theorem.

In the following theorem, we extend the necessary part of Theorem A to equation (11) without assuming that $f$ is non-decreasing or satisfies Lipschitz condition on the given interval as in [14].

Theorem 3. With respcet to the difference equation (1) assume that

$$
\sum_{n=n_{0}}^{\infty}(n+1) q_{n}<\infty
$$

Then equation (1) has a bounded nonoscillatory solution.

Proof. Set $M=\max \left\{f(x): \frac{2}{3}(1-p) \leq x \leq \frac{4}{3}\right\}$. By (23), we can choose an integer $N>n_{0}$ sufficiently large such that $M \sum_{n=N}^{\infty}(n+1) q_{n}<\frac{1-p}{3}$. Let $\mathcal{B}$ be the set of all real sequences $x=\left\{x_{n}\right\}_{n=N}^{\infty}$ with the norm $\|x\|=\sup _{n \geq N}\left|x_{n}\right|<\infty$. Then $\mathcal{B}$ is a Banach space. We define a closed, bounded and convex subset $\mathcal{S}$ of $\mathcal{B}$ as follows:

$$
\mathcal{S}=\left\{x=\left\{x_{n}\right\} \in \mathcal{B}: \frac{2(1-p)}{3} \leq x_{n} \leq \frac{4}{3}, n \geq N\right\}
$$

Define two maps $\mathcal{T}_{1}$ and $\mathcal{T}_{2}: \mathcal{S} \rightarrow \mathcal{B}$ as follows:

$$
\mathcal{T}_{1} x_{n}=\left\{\begin{array}{l}
1-p+p_{n} x_{n-k}, \quad n \geq N+\theta \\
\mathcal{T}_{1} x_{N+\theta}, \quad N \leq n \leq N+\theta
\end{array}\right.
$$




$$
\mathcal{T}_{2} x_{n}=\left\{\begin{array}{l}
-\sum_{s=n}^{\infty}(s-n+1) q_{s} f\left(x_{s-\ell}\right), \quad n \geq N+\theta \\
\mathcal{T}_{2} x_{N+\theta}, \quad N \leq n \leq N+\theta .
\end{array}\right.
$$

First we show that for any $x, y \in \mathcal{S}, \mathcal{T}_{1} x+\mathcal{T}_{2} y \in \mathcal{S}$. Infact, for every $x, y \in \mathcal{S}$ and $n \geq N+\theta$, we have

$$
\mathcal{T}_{1} x_{n}+\mathcal{T}_{2} y_{n} \leq 1-p-\frac{4}{3} p+\frac{1-p}{3}=\frac{4}{3}
$$

and

$$
\mathcal{T}_{1} x_{n}+\mathcal{T}_{2} y_{n} \geq 1-p-\frac{1-p}{3}=\frac{2(1-p)}{3} .
$$

Hence

$$
\frac{2(1-p)}{3} \leq \mathcal{T}_{1} x_{n}+\mathcal{T}_{2} y_{n} \leq \frac{4}{3} \text { for all } n \geq N .
$$

Thus, we have proved that $\mathcal{T}_{1} x+\mathcal{T}_{2} y \in \mathcal{S}$ for any $x, y \in \mathcal{S}$.

Next we shall show that $\mathcal{T}_{1}$ is a contraction mapping on $\mathcal{S}$. Indeed for any $x, y \in \mathcal{S}$ and $n \geq N+\theta$, we have

$$
\left|\mathcal{T}_{1} x_{n}-\mathcal{T}_{1} y_{n}\right| \leq p_{n}\left|x_{n-k}-y_{n-k}\right| \leq p\|x-y\| .
$$

This implies that

$$
\left\|\mathcal{T}_{1} x-\mathcal{T}_{1} y\right\| \leq p\|x-y\| .
$$

Since $p \in(0,1)$, we conclude that $\mathcal{T}_{1}$ is a contraction mapping on $\mathcal{S}$.

Now we show that $\mathcal{T}_{2}$ is completely continuous. First we will show that $\mathcal{T}_{2}$ is continuous. Let $x^{(i)}=\left\{x_{n}^{(i)}\right\} \in \mathcal{S}$ be such that $x_{n}^{(i)} \rightarrow x_{n}$ as $i \rightarrow \infty$. Because $\mathcal{S}$ is closed $x=\left\{x_{n}\right\} \in \mathcal{S}$. For $n \geq N+\theta$, we have

$$
\left|\mathcal{T}_{2} x_{n}^{(i)}-\mathcal{T}_{2} x_{n}\right| \leq \sum_{s=N+\theta}^{\infty}(s-n+1) q_{s}\left|f\left(x_{s-\ell}^{(i)}\right)-f\left(x_{s-\ell}\right)\right| .
$$

Since

$$
q_{s}(s-n+1)\left|f\left(x_{s-\ell}^{(i)}\right)-f\left(x_{s-\ell}\right)\right| \leq 2 M(s+1) q_{s}
$$

and $\left|f\left(x_{s-\ell}^{(i)}\right)-f\left(x_{s-\ell}\right)\right| \rightarrow 0$ as $i \rightarrow \infty$, in view of (23), and applying the Lebesgue dominated convergence theorem, we conclude that $\lim _{i \rightarrow \infty}\left\|\mathcal{T}_{2} x^{(i)}-\mathcal{T}_{2} x\right\|=0$. This means that $\mathcal{T}_{2}$ is continuous.

Next, we shall show that $\mathcal{T}_{2} \mathcal{S}$ is relatively compact. For any given $\varepsilon>0$, by (23) there exists an integer $N_{1} \geq N+\theta$ such that

$$
M \sum_{s=N_{1}}^{\infty}(s+1) q_{s}<\frac{\varepsilon}{2}
$$


Then for any $x=\left\{x_{n}\right\} \in \mathcal{S}$ and $j, n \geq N_{1}$,

$$
\begin{aligned}
\left|\mathcal{T}_{2} x_{j}-\mathcal{T}_{2} x_{n}\right| & \leq \sum_{s=j}^{\infty}(s-j+1) q_{s}\left|f\left(x_{s-\ell}\right)\right|+\sum_{s=n}^{\infty}(s-n+1) q_{s}\left|f\left(x_{s-\ell}\right)\right| \\
& \leq M \sum_{s=j}^{\infty}(s+1) q_{s}+M \sum_{s=n}^{\infty}(s+1) q_{s} \\
& \leq \frac{\varepsilon}{2}+\frac{\varepsilon}{2}=\varepsilon .
\end{aligned}
$$

This means that $\mathcal{T}_{2} \mathcal{S}$ is uniformly Cauchy. Hence by [5], $\mathcal{T}_{2} \mathcal{S}$ is relatively compact. By Krasonselskii fixed point theorem [6], there is a $x=\left\{x_{n}\right\} \in \mathcal{S}$ such that $\mathcal{T}_{1} x+\mathcal{T}_{2} x=x$. Clearly $x=\left\{x_{n}\right\}$ is a bounded positive solution of equation (11). This completes the proof.

\section{Oscillation results for sublinear case}

In this section we establish conditions for the oscillation and non oscillation of equation (11) when the nonlinear function $f$ is sublinear. The function $f$ is said to be sublinear if $f$ satisfies condition (5).

Theorem 4. With respect to the difference equation (1) assume $\ell>k$ and condition (5) hold. If

$$
\sum_{n=n_{0}}^{\infty} q_{n}=\infty
$$

then every solution of equation (1) is oscillatory.

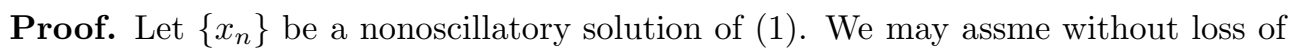
generality that $x_{n}>0$ and $0<p_{n} \leq p$ for all $n \geq N$ for some integer $N>n_{0}$. Set $y_{n}$ in (11). Using the same argument as in the proof of Theorem 2, one can consider two possible cases:

(i) $\Delta^{2} y_{n} \leq 0, \Delta y_{n}>0, y_{n}<0$ for $n \geq n_{1} \geq N+\theta$

(ii) $\Delta^{2} y_{n} \leq 0, \Delta y_{n}>0, y_{n}>0$ for $n \geq n_{2} \geq N+\theta$.

If case (i) holds, then

$$
x_{n-\ell}>-\frac{1}{p} y_{n+k-\ell}, \quad n \geq n_{1} .
$$

Substituting this into equation (11) and using the nondecreasing nature of $f(x)$, we obtain

$$
\Delta^{2} y_{n}+q_{n} f\left(-\frac{1}{p} y_{n+k-\ell}\right) \leq 0, \quad n \geq n_{1} .
$$

Summing the last inequality from $n \geq n_{1}$ to $\infty$, we find

$$
-\Delta y_{n}+\sum_{s=n}^{\infty} q_{s} f\left(-\frac{1}{p} y_{s+k-\ell}\right) \leq 0
$$


Since $-y_{n}$ in decreasing for $n \geq n_{1}$, we have from (25)

$$
-\Delta y_{n}+\left(\sum_{s=n}^{n+\ell-k} q_{s}\right) f\left(-\frac{1}{p} y_{n}\right) \leq 0 .
$$

Set $z_{n}=-\frac{1}{p} y_{n}$. Then (26) can be written as

$$
\Delta z_{n}+\frac{1}{p}\left(\sum_{s=n}^{n+\ell-k} q_{s}\right) f\left(z_{n}\right) \leq 0, \quad n \geq n_{1} .
$$

From the last inequality, it follows that

$$
\frac{\Delta z_{n}}{f\left(z_{n}\right)}+\frac{1}{p}\left(\sum_{s=n}^{n+\ell-k} q_{s}\right) \leq 0, \quad n \geq n_{1} .
$$

Summing (27) from $n_{1}$ to $N$ and using sublinear condition (5), we have

$$
\begin{aligned}
\frac{1}{p} \sum_{s=n_{1}}^{N}\left(\sum_{t=s}^{s+\ell-k} q_{t}\right) & \leq \sum_{s=n_{1}}^{N} \frac{-\Delta z_{s}}{f\left(z_{s}\right)} \\
& \leq \sum_{s=n_{1}}^{N} \int_{z_{s-1}}^{z_{s}} \frac{d u}{f(u)} \leq \int_{0}^{z_{n_{3}}} \frac{d u}{f(u)}
\end{aligned}
$$

Letting $n \rightarrow \infty$, we obtain

$$
\infty>\sum_{s=n_{1}}^{\infty}\left(\sum_{t=s}^{s+\ell-k} q_{t}\right) \geq(\ell-k) \sum_{s=n_{1}+\ell-k}^{\infty} q_{s}
$$

which contradicts condition (24) and so case (i) is impossible.

If case (ii) holds, then $x_{n} \geq y_{n}$ for $n \geq n_{2}$. Substituting this into equation (1) and using the fact thet $f(u)$ is nondecreasing in $u$, we obtain

$$
\Delta^{2} y_{n}+q_{n} f\left(y_{n-\ell}\right) \leq 0, \quad n \geq n_{2}+\theta .
$$

Summing the last inequality from $n_{3}=n_{2}+\theta$ to $\infty$, we find

$$
\sum_{n=n_{3}}^{\infty} q_{n} f\left(y_{n-\ell}\right) \leq \Delta y_{n_{3}} .
$$

Since $f$ is nondecreasing, it follows from (28) that

$$
f\left(y_{n_{2}}\right) \sum_{n=n_{3}}^{\infty} q_{n}<\Delta y_{n_{3}} .
$$


which contradicts (23) and so case (ii) is also impossible. This completes the proof of the theorem.

Theorem 5. With respect to the difference equation (1) assume that

$$
\sum_{n=n_{0}}^{\infty} f(n) q_{n}<\infty
$$

holds. Then equation (11) has an eventually positive solution which tends to infinity as $n \rightarrow \infty$.

Proof. Choose an integer $N_{0}>\theta+\frac{k}{1-p}$ sufficiently large such that

$$
\sum_{n=N_{0}}^{\infty} f(n) q_{n}<\frac{1-p}{2}
$$

Choose an integer $m>0$ such taht $m k \geq \theta$ and $N_{0}>(m+1) k$. Set

$$
a=\frac{(1-p)\left(N_{0}-m k\right)}{N_{0}-m k-p_{N_{0}-m k}\left(N_{0}-m k-k\right)} .
$$

Then

$$
1-p=\frac{(1-p)\left(N_{0}-m k\right)}{N_{0}-m k} \leq a \leq \frac{(1-p)\left(N_{0}-m k\right)}{(1-p)\left(N_{0}-m k\right)}=1 .
$$

Define the sequence $\left\{y_{n}\right\}$ as follows:

$$
y_{n}=\left\{\begin{array}{lr}
a n, & N_{0}-(m+1) k \leq n \leq N_{0}-m k \\
p_{n} y_{n-k}+(1-p) n, & N_{0}-m k \leq n \leq N_{0} \\
p_{n} y_{n-k}+(1-p) n+\sum_{s=N_{0}}^{n-1}(s-n+1) q_{s} f\left(y_{s-\ell}\right), & n \geq N_{0} .
\end{array}\right.
$$

It is easy to see that

$$
(1-p) n \leq y_{n}<n
$$

for $N_{0}-(m+1) k \leq n \leq N_{0}$. In the sequel, we prove that

$$
\frac{1}{2}(1-p) n<y_{n}<n, \quad n \geq N_{0}-(m+1) k .
$$

If (33) is not true, then there exists an integer $n_{1} \geq N_{0}$ such that

$$
y_{n_{1}} \leq \frac{1}{2}(1-p) n_{1}
$$

and

$$
\frac{1}{2}(1-p) n<y_{n}<n, \quad N_{0}-(m+1) k \leq n<n_{1}
$$


or

$$
y_{n_{1}} \geq n_{1} \text { and } \frac{1}{2}(1-p) n<y_{n}<n, \quad N_{0}-(m+1) k \leq n<n_{1} .
$$

If (34) holds, then from (30), (31) and (34), we have

$$
\begin{aligned}
y_{n_{1}} & =p_{n_{1}} y_{n_{1}-k}+(1-p) n_{1}+\sum_{s=N_{0}}^{n_{1}-1}\left(s-n_{1}+1\right) q_{s} f\left(y_{s-\ell}\right) \\
& \geq(1-p) N_{0}+\left(n_{1}-N_{0}\right)\left[1-p-\sum_{s=N_{0}}^{n_{1}-1} q_{s} f\left(y_{s-\ell}\right)\right] \\
& \geq(1-p) N_{0}+\left(n_{1}-N_{0}\right)\left[1-p-\sum_{s=N_{0}}^{n-1} q_{s} f\left(y_{s-\ell}\right)\right] \\
& >(1-p) n_{0}+\left(n_{1}-N_{0}\right)\left[1-p-\frac{1-p}{2}\right] \\
& >\frac{1}{2}(1-p) n_{1} .
\end{aligned}
$$

This contradiction implies that (34) is not true. If (35) holds then from (30), (31) and (35) we have

$$
\begin{aligned}
y_{n_{1}} & =p_{n_{1}} y_{n_{1}-k}+(1-p) n_{1}+\sum_{s=N_{0}}^{n_{1}-1}\left(s-n_{1}+1\right) q_{s} f\left(y_{s-\ell}\right) \\
& \geq p\left(n_{1}-k\right)+(1-p) n_{1}=n_{1}-p k<n_{1} .
\end{aligned}
$$

This is also a contradiction and so (35) is not true. Therefore (33) holds. It is easy to see that $\left\{y_{n}\right\}$ satisfies the equation

$$
\Delta^{2}\left(y_{n}-p_{n} y_{n-k}\right)+q_{n} f\left(y_{n-\ell}\right)=0, \quad n \geq N_{0} .
$$

This shows that $\left\{y_{n}\right\}$ is a positive solution of equation (11) with the desired asymptotic behavior. The proof is now complete.

\section{References}

[1] R. P. Agarwal, Difference Equations and Inequalities, Marcel Dekker, New York, 2000.

[2] R. P. Agarwal, S. R. Grace and D. O' Regan, Oscillation Theory for Difference and Functional Differenctial Equations, Kluwer Academic, 2000.

[3] R. P. Agarwal, M. M. S. Manuel and E. Thandapani, Oscillatory and nonoscillatory behavior of second order neutral delay difference equations, Math. Comput. Modelling 24(1996), $5-11$.

[4] M. Budericenic, Oscillation of a second order neutral difference equation, Bull Cl. Sci, Math. Nat. Sci. Math. 22(1994), 1-8. 
[5] S. S. Cheng and W. T. Patula, An existence theorem for a nonlinear difference equation, Nonlinear Anal. 20(1993), 193-203.

[6] L. H. Erbe, Q. Kong and B. G. Zhang, Oscillation Theory for Functional Differential Equations, Marcel Dekker, New York, 1995.

[7] S. R. Grace and B. S. Lalli, Oscillation theorem for second order delay and neutral difference equations, Utilitaes Math. 45(1994), 197-211.

[8] W. T. Li, Oscillation of higher order neutral nonlinear difference equations, Appl. Math. Lett. 11(1998), 1-8.

[9] H. J. Li and C. C. Yeh, Oscillation criteria for second order neutral delay difference equations, Comput. Math. Appl. 36(1998), 123-132.

[10] Z. Liu, S. Wu and Z. Zhang, Oscillation of solutions for even order nonlinear difference equations with nonlinear neutral term, Indian J. Pure Appl. Math. 34(2003), 1585-1598.

[11] A. Sternal and B. Szmanda, Asymptotic and oscillatory behavior of certain difference equations, Le Mat. 51(1996), 77-86.

[12] X. H. Tang and Y. J. Liu, Oscillations for nonlinear delay difference equations, Tamkang J. Math. 32(2001), 275-280.

[13] E. Thandapani and R. Arul, Oscillation properties of second order nonlinear neutral delay difference equations, Indian J. Pure Appl. Math. 28(1997), 1567-1571.

[14] E. Thandapani and K. Mahalingam, Necessary and sufficient conditions for oscillation of second order neutral difference equations, Tamkang J. Math. 34(2003), 137-145.

[15] E. Thandapani, P. Sundaram, J. R. Graef and P. W. Spikes, Asymptotic properties of solutions of nonlinear second order neutral delay difference equations, Dymanic Sys. Appl. 4(1995), 125-136.

[16] A. Zafer and R. S. Dahiya, Oscillation of a neutral delay difference equations, Appl. Math. Lett. 6(1993), 71-74.

[17] G. Zhang, Oscillation for nonlinear neutral difference equations, Applied Math. E- Notes $\mathbf{2}(2002), 22-24$.

[18] B. G. Zhang and S. H. Saker, Kamenev-type oscillation criteria for nonlinear neutral delay difference equations, Indian J. Pure Appl. Math. 34(2003), 1571-1584.

[19] Z. Zhang, J. Chen and C. Zhang, Oscillation of solutions for second order nonlinear difference equations with nonlinear neutral term, Comput. Math. Appl. 41(2001), 1487-1494.

[20] G. Zhang, S. S. Cheng and Y. Gao, Classification schemes for positive solutions of a second order nonlinear difference equations, J. Comp. Appl. Math. 101(1999), 39-51.

Department of Mathematics, Periyar University, Salem - 636 011. Tamilnadu, India.

E-mail: ethandapani@yahoo.co.in

Department of Mathematics, Periyar University, Salem - 636 011. Tamilnadu, India. 